\title{
Impact testing of polymeric foam using Hopkinson bars and digital image analysis
}

\author{
Jiagui Liu ${ }^{\mathrm{a}}$, Dominique Saletti ${ }^{\mathrm{b}}$, Stéphane Pattofatto ${ }^{\mathrm{c}}$, Han Zhao ${ }^{\mathrm{a}, *}$ \\ ${ }^{a}$ Laboratoire de Mécanique et Technologie (LMT-Cachan), ENS-Cachan/CNRS-UMR8535/Université Pierre 83 \\ Marie Curie, 61, avenue du président Wilson, 94235 Cachan cedex, France \\ ${ }^{b}$ Laboratoire de Biomécanique (LBM), Arts et Mtiers ParisTech, Paris, France \\ ${ }^{c}$ Snecma Villaroche, Safran Group, Rond Point René Ravaud-Réau, 77550 Moissy-Cramayel, France
}

\begin{abstract}
This paper has investigated the impact testing method on the polymeric foams using digital image correlation. Accurate average stress-strain relations can be obtained when soft large diameter polymeric pressure bars and appropriate data processing are used. However, as there is generally no homogeneous strain and stress fields for polymeric foam, an optical displacement field observation is essential. In contrast with quasi-static tests where the digital image correlation (DIC) measurement is commonly used, technical difficulties still remain for an use under impact conditions such as synchronization and measuring accuracy due to rather poor quality images obtained from high speed camera. In the present paper, an accurate synchronization method based on direct displacement measurement from DIC is proposed and the feasibility of a calibration method using DIC on pressure bar ends is discussed. The relevance of the present method for establishing mechanical response of polymeric foam is also demonstrated.
\end{abstract}

Keywords: Polymeric foam, Impact testing, Hopkinson bars, High-speed imaging, Digital image correlation.

\section{Introduction}

Polymeric foams have been widely used in weight-saving and energy-absorbing structural applications, such as automobile vehicles, aircraft structures [1] and personal protections [2]. Under quasi-static loading, analytical/numerical approaches were developed to give a reasonable prediction of foam-like material behaviors [3, 4, 5, 6, 7] and experimental data were obtained without difficulties. However, such approaches cannot be easily generalized in the case of impact

\footnotetext{
* Corresponding author.

Email address: zhao@lmt.ens-cachan.fr (Han Zhao)
} 
loading, which is generally the real working conditions of polymeric foams for energy absorption applications. It is therefore very important to develop a dynamic experimental approach to characterize polymeric foams under impact loading.

The behavior of polymeric foams at relatively high strain rates has been an interest of investigators since 1960s [8, 9]. Experimental results using different devices such as the falling weight or impacting mass technique [10, 11, 12], rapid hydraulic testing machine [13, 14, 15] and also split Hopkinson bar have been reported [16, 17, 18]

Hopkinson bars have been widely used to experimentally characterize the mechanical behaviors of materials under impact strain rates loading, from $10^{2} \mathrm{~s}^{-1}$ to $10^{4} \mathrm{~s}^{-1}$ and provide more accurate measurement than drop weight and high-speed testing machine at this range. Common metallic bar with quartz-crystal piezoelectric force transducers [18] or large diameter nylon pressure bars [19, 20] were used to improve signal/noise ratio. Typical overall stress-strain curves at high strain rates were obtained with such nylon pressure bars method for polymeric foams [21] and metallic foams [22].

Meanwhile, it is noticed that aforementioned testing results (under static as well as impact loading rate) are only the average stress-strain relations with an assumption of homogeneous stress and strain fields within the foam specimen, which is unfortunately not true for most of the foam-like materials. This non-uniform or even localized deformation has been observed by a number of studies [7, 19, 23].

Thus, field observation techniques have been added in more recent tests, often associated with digital image correlation (DIC) [24, 25]. In contrast with rather common applications of photography and DIC to the foam testing under static loading [26, 27, 28], only a few preliminary studies with high speed imaging were reported under impact loading rates [29, 30, 31, $32, \underline{33}$ ] because of synchronization difficulties and poor image resolution from high-speed camera.

In this paper, an optical field measurement along with large diameter nylon Hopkinson bars is highlighted in the experimental setup for the polymeric foams characterization. Nylon Hopkinson bar methods and the associated data processing are briefly recalled in the section 2. Afterwards, technical points of high speed strain field measurement such as time synchronization accuracy as well as DIC method with poor resolution images is discussed. Finally, testing of a polymeric foam is given as an example to illustrate the needs of an accurate field measurement. 


\section{Conventional tests on the polymeric foams}

\subsection{Foam specimen and quasi-static tests}

Conventional quasi-static compressive tests on polymeric foams can be performed using a universal testing Machine. A polymeric foam supplied by EADS company is studied as an example. The density is $70 \mathrm{~kg} / \mathrm{m}^{3}$, the diameter of unit cell is about $0.8 \mathrm{~mm}$ and the thickness of the cell wall is about $50 \mu \mathrm{m}$ from microscopy observations. The specimen tested in this study is a cylinder of $60 \mathrm{~mm}$ in diameter and $40 \mathrm{~mm}$ in height.

MTS 810 material testing system is used. The specimen is centrally sandwiched between the two platens. The lower one rises at a constant speed of $0.2 \mathrm{~mm} / \mathrm{s}$ during loading, which ensures the nominal strain-rate of $5 \times 10^{-3} \mathrm{~s}^{-1}$. Three tests are carried out and repeatability is checked (see in Fig. 10. This mechanical response is typical for cellular materials which exhibits a linear

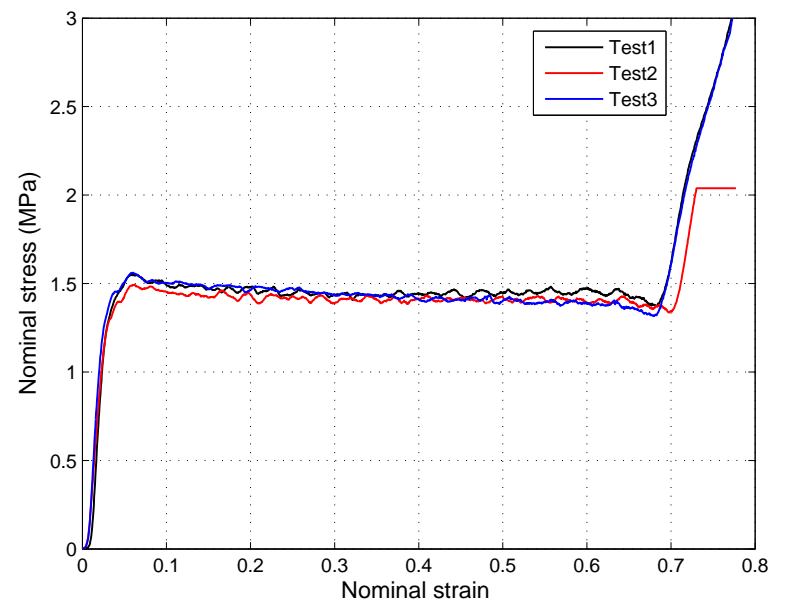

Figure 1: Three nominal stress - nominal strain curves from quasi-static loading

increase or elastic period, followed by a very long plateau and a densification phase.

\subsection{Impact tests with $S H P B$}

The dynamic tests are performed on nylon Hopkinson bar. A typical SHPB is made of long input and output pressure bars with a short specimen sandwiched between them. The impact between the projectile and the input bar generates a compressive longitudinal incident pulse $\varepsilon_{i}(t)$ in the input bar. Once this incident pulse reaches the specimen-bar interface, one part is reflected as the reflected pulse $\varepsilon_{r}(t)$ in the input bar and the other part transmitted as the transmitted 
pulse $\varepsilon_{t}(t)$ in the output bar. With the gauges glued on the input bar and output bar, one can record these three pulses.

The transmitted wave can be shifted to the output bar-specimen interface to obtain the output force and velocity, at the same time, the input force and velocity can be determined via incident and reflected waves shifted to the input bar-specimen interface. Forces and velocities at both faces of the specimen are then given by the following:

$$
\begin{array}{ccc}
F_{\text {input }}=S_{B} E\left(\varepsilon_{i}(t)+\varepsilon_{r}(t)\right), \quad \text { and } & V_{\text {input }}=C_{0}\left(\varepsilon_{i}(t)-\varepsilon_{r}(t)\right), \\
F_{\text {output }}=S_{B} E\left(\varepsilon_{t}(t)\right) & V_{\text {output }}=C_{0} \varepsilon_{t}(t)
\end{array}
$$

where $F_{\text {input }}, F_{\text {output }}, V_{\text {input }}, V_{\text {output }}$ are forces and particle velocities at the input and output interfaces, $S_{B}, E$ and $C_{0}$ are the cross-sectional area, Young's modulus and longitudinal wave speed in the pressure bars respectively. $\varepsilon_{i}(t), \varepsilon_{r}(t)$ and $\varepsilon_{t}(t)$ are the strain signals at the barspecimen interfaces.

SHPB system used for this study consists of two $3 \mathrm{~m}$ long nylon bars of $62 \mathrm{~mm}$ diameter (density $1200 \mathrm{~kg} / \mathrm{m}^{3}$ and wave speed $1800 \mathrm{~m} / \mathrm{s}$ ). The use of the nylon bars multiply by 20 the impedance ratio between the foam and the bar material(thus the signal/noise ratio), in comparison with a classical steel bar (density being $7850 \mathrm{~kg} / \mathrm{m}^{3}$ and wave speed $5000 \mathrm{~m} / \mathrm{s}$ ). However, with the use of big diameter visco-elastic bars, the reasoning in Eq. (1) should be amended by considering wave dispersion effect in the shift from measuring points to the bar/specimen interfaces 34, 35]. The correction of this dispersion effect on the basis of a generalized Pochhammer's wave propagation theory is consequently performed in data processing [19].

As the coefficients between the strain and recorded tension may be influenced by many factors, such as gauges factors, temperature, humidity or gain of the amplifier, calibration of these coefficients is very important for the overall precision. The principle of calibration for the input bar is on the basis of the energy conservation, i.e., the kinetic energy of the impactor is equal to the sum of the kinetic energy and the elastic energy carried by the incident impulse in the input bar. This is valid if the input bar and the impactor have the same properties (diameter, material).

The calibration of the output bar is realized by the force balance during a test without specimen. Here, the forces can be calculated as Eq. (1) and should be exactly the same because they indicate the force at the same interface (input bar/output bar interface). Therefore, the calibration coefficient for the gauge on the output bar is ascertained.

From the forces and velocities at both bar-specimen interfaces, nominal stress and nominal 
strain can be obtained following the standard formulas of SHPB analysis, which assumes the uniformity of stress and strain fields:

$$
\begin{gathered}
\sigma_{s}(t)=\frac{F_{\text {output }}}{S_{s}} \\
\varepsilon_{s}(t)=\int_{0}^{t} \frac{V_{\text {output }}(\tau)-V_{\text {input }}(\tau)}{l_{s}} \mathrm{~d} \tau
\end{gathered}
$$

The present Hopkinson bars experiments followed this procedure with wave dispersion correction and gauge calibrations. Before every test, the interfaces of bar-specimen are lubricated to decrease the frictional effect as much as possible. Input and output forces history is examined to check whether the dynamic equilibrium is achieved. An example is given in Fig. 2 for a test at $10 \mathrm{~m} / \mathrm{s}$. The equilibrium is established around $0.3 \mathrm{~ms}$.

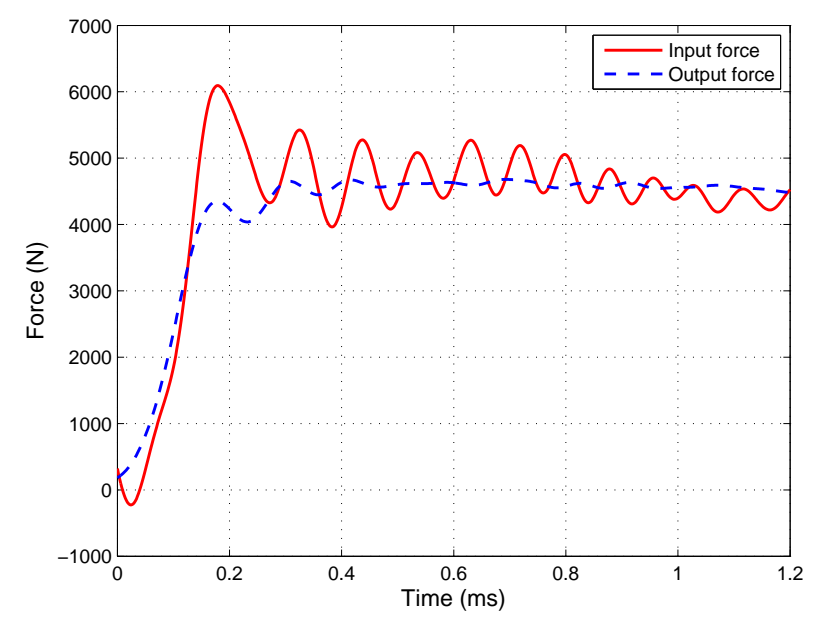

Figure 2: Forces history at specimen interfaces for a test at $10 \mathrm{~m} / \mathrm{s}$. Force equilibrium is checked.

The mechanical response of the foam at $18 \mathrm{~m} / \mathrm{s}$ is represented in Fig. 3 and is compared to the quasi-static case. One can notice that, for dynamic loading, the densification phase is not reached, due to the impulse length generated in the SHPB system.

Following the same procedure, seven specimens are tested using nylon SHPB with the projectile speeds ranging from $7 \mathrm{~m} / \mathrm{s}$ to $19 \mathrm{~m} / \mathrm{s}$. The plateau stress remains nearly constant in the range of impact loading speeds (Fig. (4), from $5 \times 10^{-3} \mathrm{~s}^{-1}$ to $4.5 \times 10^{2} \mathrm{~s}^{-1}$. However the average strength increased of $8 \%$ from quasi-static loading to impact loading. 


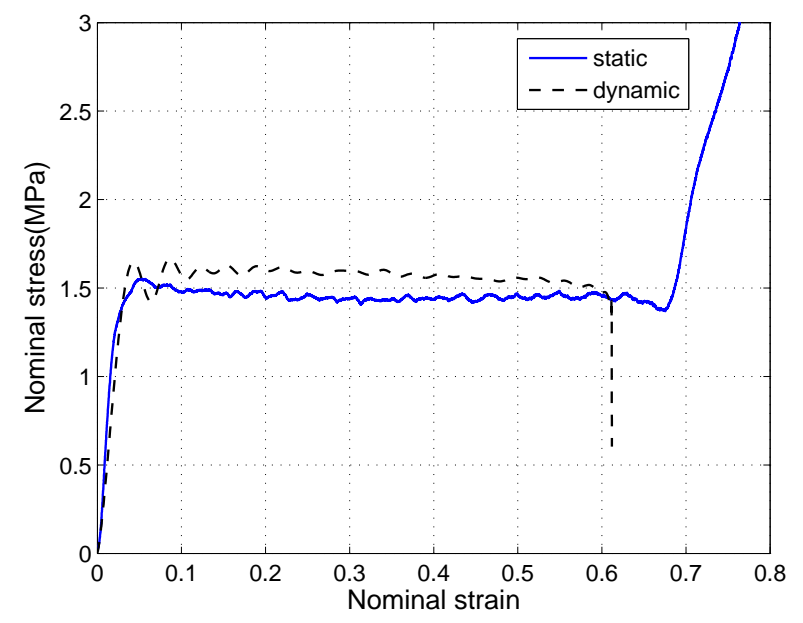

Figure 3: Comparison of nominal stress - nominal strain behavior under impact loading and quasi-static loading

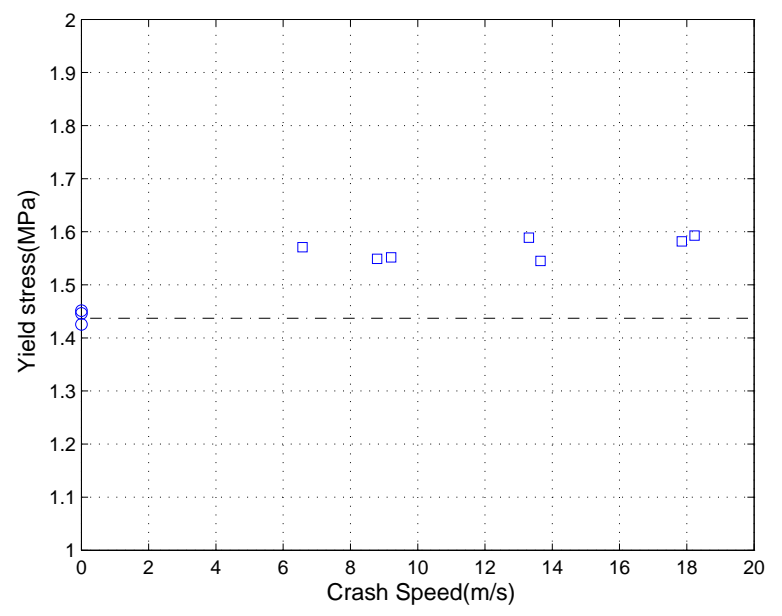

Figure 4: Mean plateau stress vs impact speed under both dynamic and quasi-static loading 


\section{Digital photography and image correlation}

As mentioned in the introduction, digital photography and the associated digital image correlation is nowadays widely used to observe the deforming mode of the specimen, to monitor the loading condition and to calculate the strain field. Even though the equilibrium state within the specimen is reached, heterogeneous strain field is still possible with a localized area such as the case of honeycombs for example.

\subsection{Direct image observations}

For quasi-static loading, a Canon EOS 50D camera is used and triggered with an equal timeinterval signal $(3.5 \mathrm{~s})$. Fig. 5 shows the deforming states of the specimen at different crush displacements. The deformation of the foam is highly localized in the darker areas, visible next to the platens.

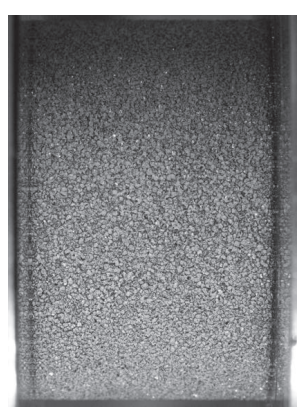

$\mathrm{d}=4.01 \mathrm{~mm}$

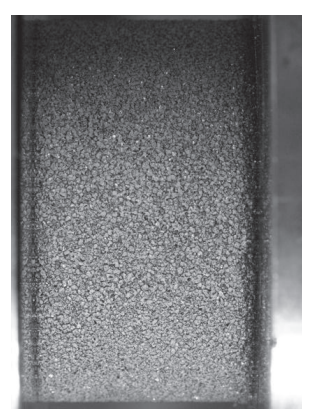

$\mathrm{d}=7.55 \mathrm{~mm}$

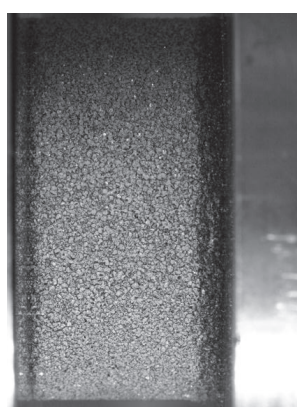

$\mathrm{d}=11.78 \mathrm{~mm}$

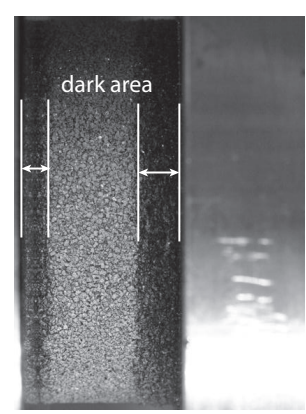

$\mathrm{d}=19.53 \mathrm{~mm}$

Figure 5: The deforming states at different crush displacements under quasi-static loading

A simple image processing is made with such images. By locating the boundary of the darker area, the length of the uncollapsed part can be obtained, which is plotted in Fig. 6. The $x$-axis is the actual specimen length obtained by subtracting the prescribed crush displacement from initial length of the specimen. In this figure, the markers $*$ are corresponding respectively to the last three images of Fig. 5 (the darker band is too small to be processed on the first image). It can be seen that the length of the uncollapsed part decreases linearly.

One can find that the strain is highly localized in the collapsed areas and the crush displacement is mainly obtained by the increase of these areas. Along with the stress-strain curve in Fig. 3, one may conclude that the specimen collapses little by little at the ends as long as the crush displacement goes and it thus manifests a plateau in the stress-strain curve. Whether this 
conclusion is correct or not, it needs further examination of the strain field on the surface of the specimen.

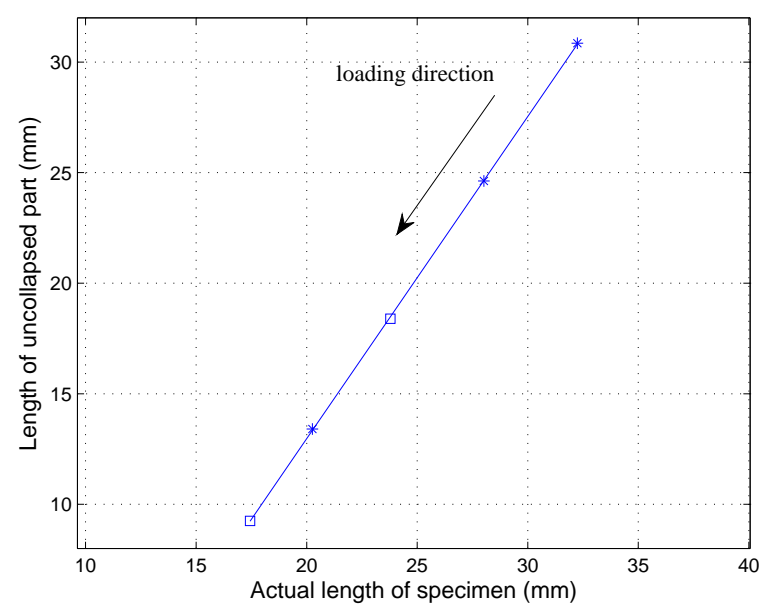

Figure 6: Evolution of the uncollapsed central part length with the current length of specimen, where the stars mark the values corresponding to the last three images of Fig. 5

\subsection{Principle of digital image correlation}

More sophisticated image processing can be used to match the material points in the image sequence and to calculate displacement fields. Consequently, a quantitative strain fields can be offered.

DIC code Correli ${ }^{4}$, developed in LMT-Cachan is used. It is a global approach of image correlation based on the conservation of optical flow. Considering a reference image $f$ and a deformed image $g$, the conservation of optical flow can be written as (in 1D for brevity):

$$
f(x)=g[x+u(x)]
$$

where $u(x)$ is the motion from the reference image to the deformed image. When the motion is small, the first order Taylor expansion of $g[x+u(x)]$ yields to:

$$
g[x+u(x)] \approx g(x)+g^{\prime} \cdot u(x)
$$

To measure the motion $u(x)$, a minimization of the gray level residual is therefore introduced on the region of interest $(\mathrm{ROI})$ :

$$
\eta^{2}=\int_{R O I}\{f(x)-g[x+u(x)]\}^{2} \mathrm{~d} x
$$


Assuming that the motion $u(x)$ is expressed in a space of basis functions, $\left\{\psi_{p}\right\}$, i.e.

$$
u(x)=\sum u_{p} \psi_{p}(x)
$$

where $u_{p}$ is the unknown degrees of freedom. By substituting Eq. (6) to Eq. (5), it thus obtains:

$$
\eta^{2}=\int_{R O I}\left\{f(x)-g\left[x+\sum u_{p} \psi_{p}(x)\right]\right\}^{2} \mathrm{~d} x
$$

And considering the first order approximation, Eq. (4), the minimization of the object function becomes:

$$
\eta_{l i n}^{2}=\int_{R O I}\left[f(x)-g(x)-\sum u_{p} \psi_{p}(x) g^{\prime}(x)\right]^{2} \mathrm{~d} x
$$

An iterative scheme is followed to obtain the best estimation of $u_{p}$, and thus $u(x)$. Furthermore, the strain field in the ROI is obtained $([25,36,37])$. In this case, Q4P1-shape functions are chosen as the basis functions so that the measured displacement field is consistent with FE analysis computations.

\subsection{Strain field analysis}

Displacement and strain fields on the surface of the specimen are calculated by DIC. Only the uncollapsed part is chosen as ROI. Indeed, the highly densified dark zone leads to convergency problem in DIC algorithm because the gradient of gray is nearly zero in this area. It might be worth noting that $2 \mathrm{D}$ image correlation generally requires the object moving in a plane parallel to the camera sensor, which is not the case with the present cylindrical specimen. Nevertheless, the strain in transversal direction is not our interest here. Moreover, as Poisson ratio is low, transversal strain is limited and does not affect the longitudinal strain. As a result, the strain analysis with DIC on the cylindrical specimen is still acceptable to provide the longitudinal strain.

32 pixels $\times 32$ pixels element size is adopted for quasi-static cases. The ROI and the strain fields are shown in Fig. 7 in which the strain fields correspond to the images in Fig. 5, The results show the non-uniformity of strain even within this apparently uncollapsed part.

It might be interesting to compare the nominal strain with the DIC strain measurement of this uncollapsed part of specimen. The evolutions of the global nominal strain and the average value of the DIC strain are plotted with respect to the image number in Fig. 8. Great disparity is found between these two curves. The nominal strain increases rather linearly and the average local strain remains constant at a value of about $2.2 \%$. The DIC strain measurement confirms that a localized deformation area is created when the specimen is compressed to a critical strain around $2.2 \%$. 


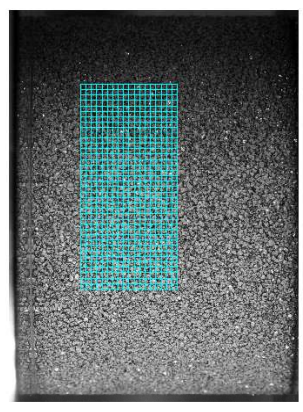

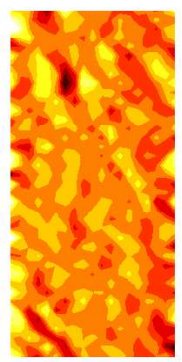

$\mathrm{d}=4.01 \mathrm{~mm}$

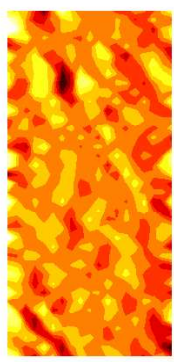

$\mathrm{d}=7.55 \mathrm{~mm}$

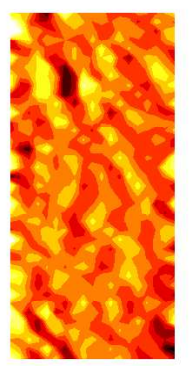

$\mathrm{d}=11.70 \mathrm{~mm}$

0.005
-0.000
-0.006
-0.011
-0.017
-0.023
-0.028
-0.034
-0.039
-0.045
-0.050

Figure 7: ROI calculated in quasi-static loading and the longitudinal strain fields

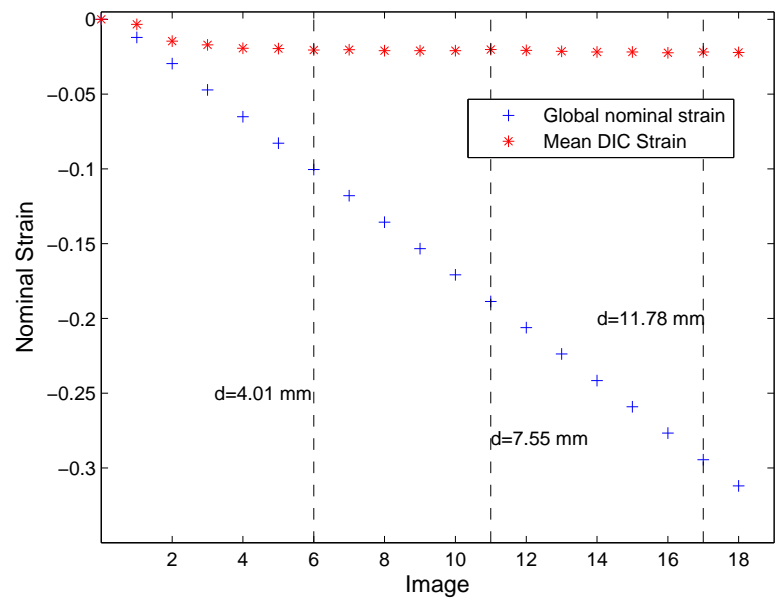

Figure 8: Comparison of the global nominal strain and the mean local strain in the quasi-static loading 


\section{Field measurement under impact loading}

\subsection{High Speed digital image system}

For the Hopkinson bar tests, a Photron Fastcam SA5 high-speed camera is used to record the deforming process of the specimen during impact tests. Image sequences with a 384 pixels $\times 432$ pixels resolution at 40,000 $\mathrm{fps}$ is acquired. Shutter speed is set to $1 / 70,000 \mathrm{~s}$ and aperture f5.6 so that the obtained images are sharp enough. Thanks to two powerful cold spotlights, the images are ensured to a suitable contrast in despite of the small aperture, high shutter speed and frequency.

Compared to the static case, there are no apparent dark areas in the image sequences from dynamic experiments. An example is given in Fig. 9 for a prescribed velocity of $10 \mathrm{~m} / \mathrm{s}$. Instead, a lot of fragments and localized bands are found.

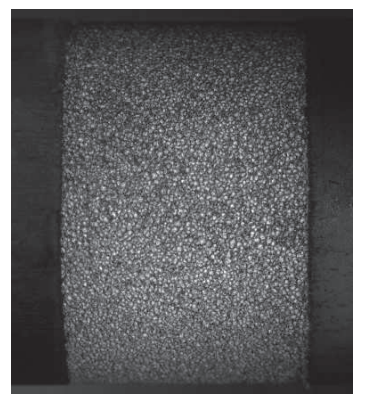

$\mathrm{d}=1.99 \mathrm{~mm}$

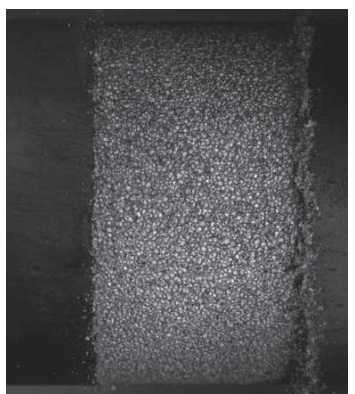

$\mathrm{d}=7.57 \mathrm{~mm}$

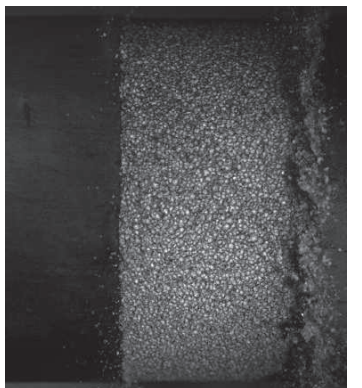

$\mathrm{d}=11.82 \mathrm{~mm}$

Figure 9: The deforming states at different crush displacement under impact loading

The synchronization between images and SHPB signals is not as natural as in quasi-static tests. Hopkinson bars and high speed camera, which are independent for conventional experiments, are connected with a multi-channel data link (MCDL) box as shown in Fig. 10, In practice, a LabView program has been created and records the signals from the strain gauges on the input bar and the output bar once triggered. At the same time it sends a TTL signal of 5 volts to trigger the high speed camera. For the MCDL, only one signal from Hopkinson bar system is sampled, with a frequency equal to 10 times the camera frame rate. This sample signal is sent to camera and stored in a common file with the image number shot at the same instant. This file is the basis of the synchronization between the Hopkinson bar system and the high speed camera system. 


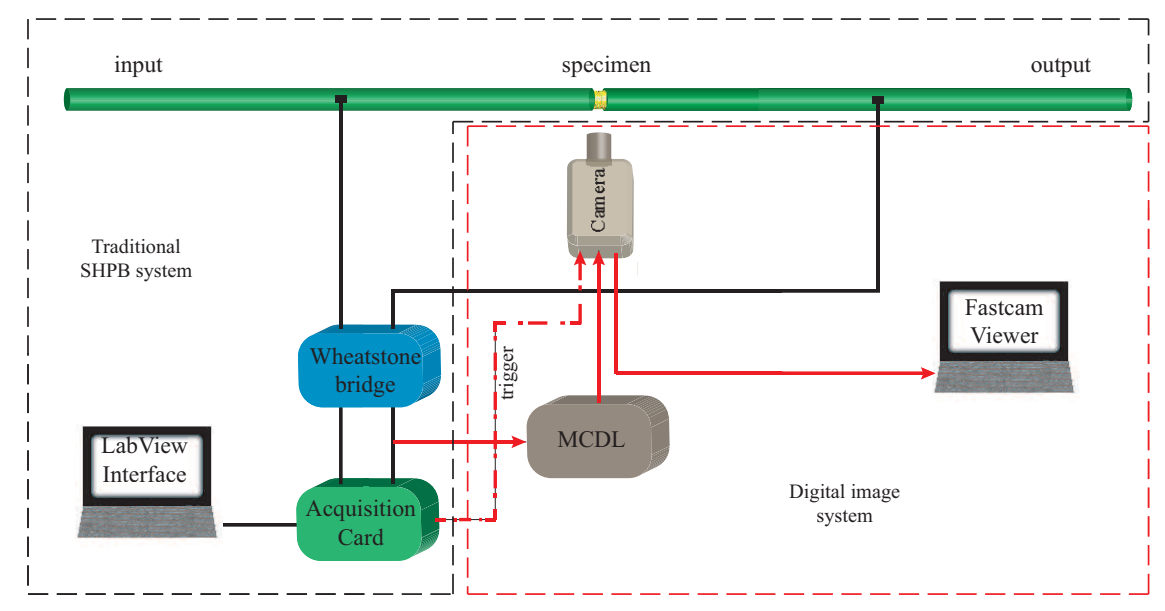

Figure 10: Diagram of SHPB system and the digital image photography system

\subsection{Synchronization experimental results and images}

As indicated before, the MCDL box samples the signal from the gauge on the output bar, named $v$. The same signal recorded by the acquisition card with different acquisition frequency, $500 \mathrm{KHz}$ in our case, is named $u$. The two signals should coincide exactly on time because they represent the same physical event. Therefore, synchronization between stress-strain curve and images can be established (see the diagram in Fig. 11).

Indeed, as $u$ and $v$ share the same source, synchronization could be established by shifting them in time axis. The procedure is as following: 1) interpolating one of them acquired with lower frequency to the higher one, i.e. making sure that the two series have same time interval between two adjacent points, $u(i)$ and $v(i)$ are the interpolated results; 2) make a convolution between $u(i)$ and $v(i)$, and find the maximum location of convolutional result. The convolution is realized in detail as:

$$
w(k)=\sum_{j} u(j) \cdot v(n-k+j)
$$

where $n$ is the length of series $v(i)$. The maximum convolutional result, $w(k)$, is corresponding to the location where $u(i)$ and $v(i)$ are best superposed and the time is thus needed to shift in order to achieve the synchronization. 


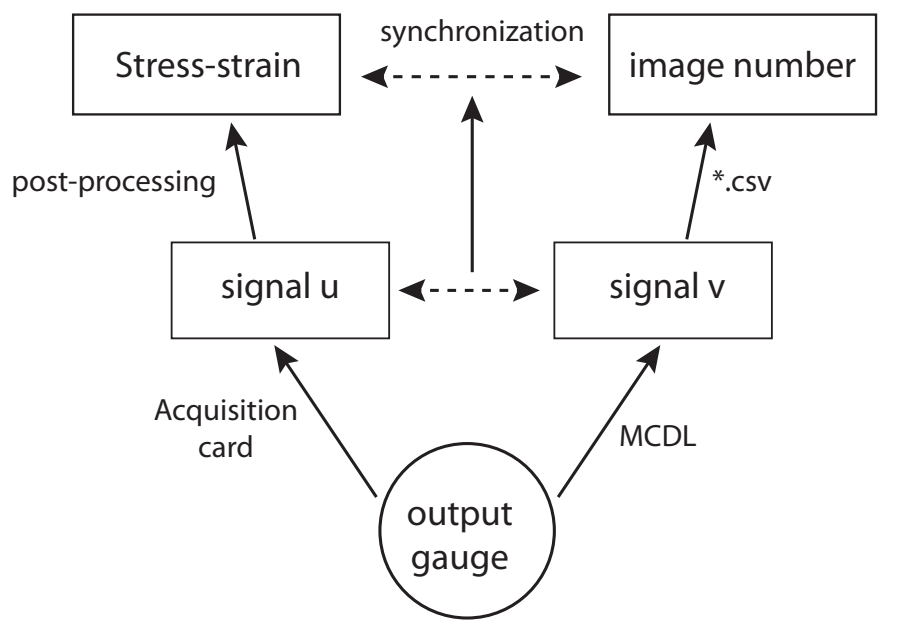

Figure 11: Diagram of the relationship between stress-strain curve and images via MCDL

\subsection{Tracking the movement of the bar}

If this synchronization is accurate and there is no time shift error from strain signals in the pressure bar, the displacements of the bars calculated from SHPB signals by integrating the velocities in Eq. (11) will be equal to the displacements from DIC measurement. It thus provide a possibility to check the accuracy of aforementioned synchronization method as well as calibration of strain/tension coefficient (position and scale respectively). Therefore, the ends of pressure bars are also captured during every test (see in Fig. 12) and DIC will use them to calculate the displacements of the bars.

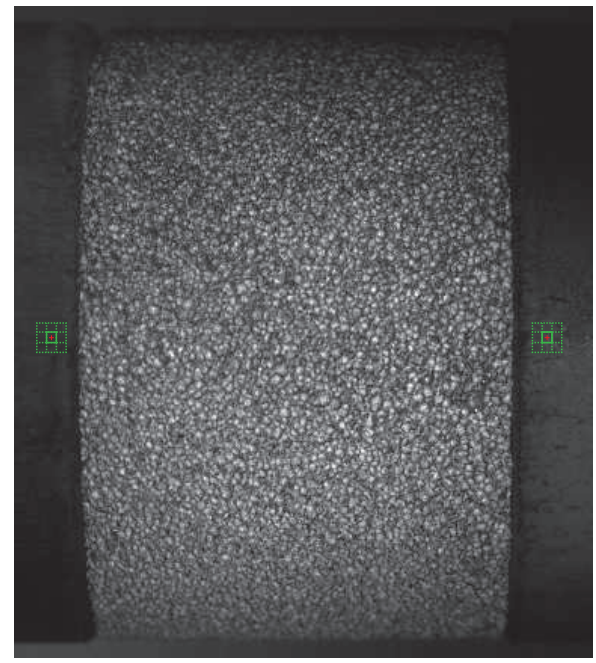

Figure 12: The illustration of the points on the bars traced by DIC 
To trace the displacement of one point on the bar (the cross located in the central element in Fig. 12), a small element of 8 pixels $\times 8$ pixels (about $1 \mathrm{~mm} \times 1 \mathrm{~mm}$ in reality) is adopted to calculate the displacement of the bar (see the solid square in Fig. 12). The 8 surrounding elements help for this calculation. It is stressed that the strain of the small element on the bar is ignored, thus the displacement of the bar is calculated by averaging displacements of its four nodes. Generally, the point traced is very close to the bar-specimen interface, usually less than $2 \mathrm{~mm}$. Therefore, the displacement of the bar in this point is regarded as the displacement of the interface. It is worthwhile to note that when the strain can be accurately measured in the bars with camera technical evolution, a direct stress/force measurement at the ends of the bars will be available.

\subsection{Validation of synchronization}

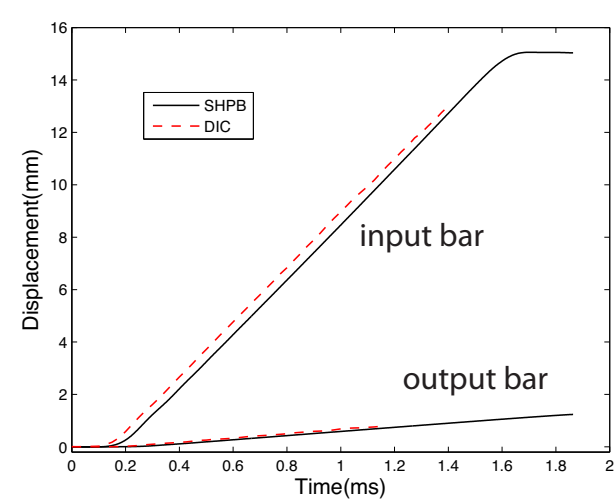

a

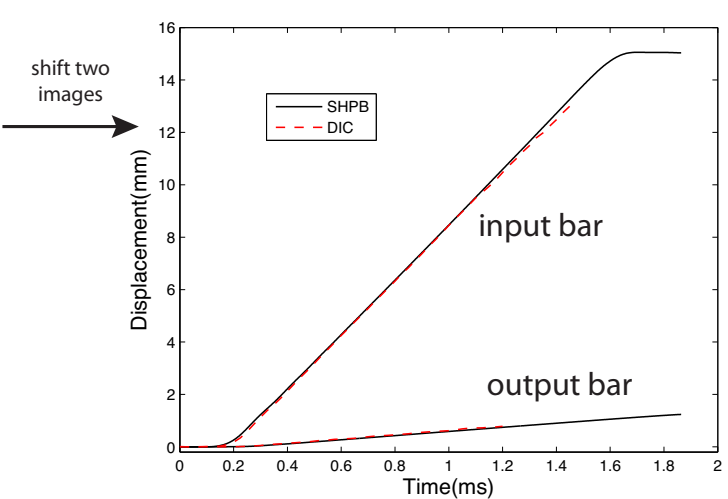

b

Figure 13: Comparison of displacements-time profiles obtained by SHPB and DIC tracking method: a) synchronized directly using the method (MCDL) detailed in section 4.2 (b) two images shifted to make coincidence.

The synchronization method introduced in section 4.2 gives a correspondance between images and experimental data from Hopkinson bars. Therefore, the displacements of both specimen-bar interfaces synchronized are plotted in Fig. 13(a). The two solid lines are the displacements of bar-specimen interfaces measured by SHPB, while the dash lines are the displacements calculated with DIC tracking method (section 4.3). A significant gap is observed in the displacements at the input end, the displacement at the output end is too small to observe an obvious gap. However, the superpositions are well achieved if two images shift on the basis of the synchronization by MCDL method, as shown in Fig. 13(b). 
Consequently, the conclusion can be made as that: firstly, both the displacements from SHPB and DIC tracking method are credible because of the possibility of good superpositions, there is no error of the coefficient calibrations, nor DIC parameter mistake; secondly, the synchronization method with MCDL box is not very accurate. In fact, the delay of several images in synchronization exists in all the experiments, not only in Nylon pressure bars presented in this paper but also in tensile bars in our laboratory. As a result, the examination of synchronization with DIC analysis is necessary in the case of high speed camera.

\subsection{DIC measurements}

For the uncollapsed part of the specimen, the DIC measurement is possible. In contrast to static case, elements size of 8 pixels $\times 8$ pixels $(1 \mathrm{~mm} \times 1 \mathrm{~mm}$ in reality $)$ are employed in DIC algorithm. The chosen ROI along with the undeformed reference image is shown in Fig. 14, These strain fields corresponding to the images shown in Fig. 9are also presented in Fig.14. They manifest that even the uncollapsed part of the specimen is not compressed uniformly because each of these three strain fields shows color difference or color strips. Some highly deformed spots scatter in the fields, which reveals that some cells are largely compressed or collapsed. Moreover, the color difference becomes larger for image from left to right (from small crush displacement to the large one) and the number of the highly deformed spots increases as well.

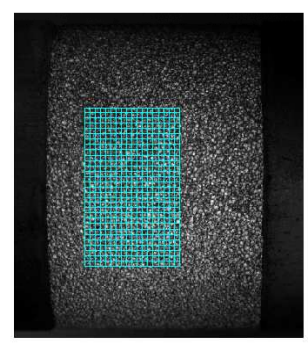

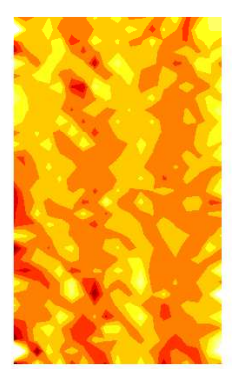

$\mathrm{d}=1.99 \mathrm{~mm}$

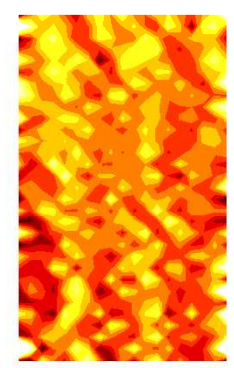

$\mathrm{d}=7.57 \mathrm{~mm}$

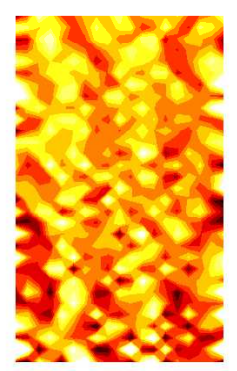

$\mathrm{d}=11.82 \mathrm{~mm}$

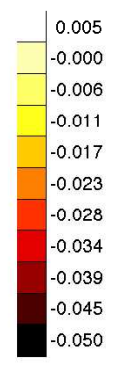

050

Figure 14: ROI calculated in impact loading and the longitudinal strain fields which are corresponding to the images in Fig. 9

Similarly to the quasi-static case, the global nominal strain and average value of the DIC local strain are compared, see in Fig. 15. The same conclusion can be made as the quasi-static one while the plateau strain is about $1.9 \%$. 


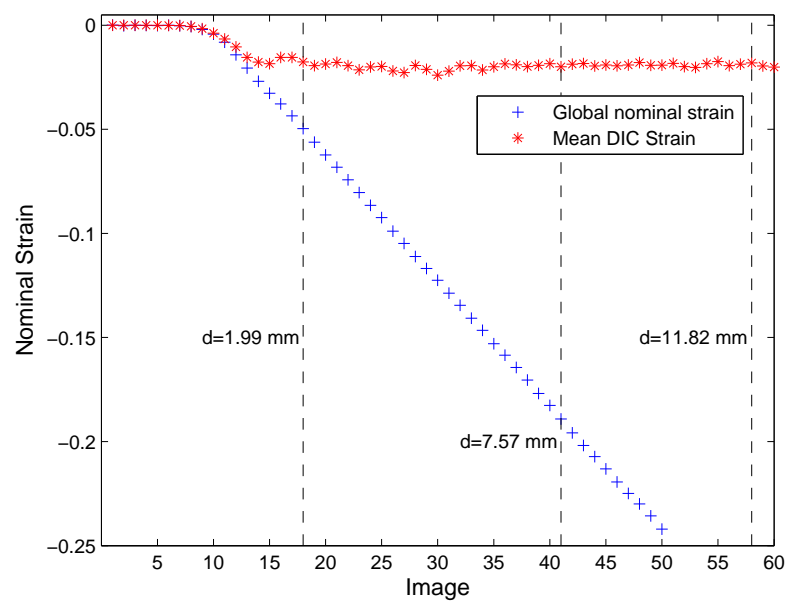

Figure 15: Comparison of the global nominal strain and the mean local strain in the dynamic loading

With the knowledge of both the strain localization feature and the plateau of the mean local strain, the real deforming process of this polymeric foam is unveiled. At the beginning of the loading, the foam is firstly compressed as a whole where the stress increases linearly. With the increase of the strain, the limit of failure stress of cells is reached and they begin to collapse. From then on, the collapse front propagates while the uncollapsed part stops being further compressed, which displays the plateaus in the mean local strain and in nominal stress.

\section{Conclusion}

This paper deals with impact testing on the polymeric foams using large diameter nylon Hopkinson bar technique and optical DIC strain field measurement. Even though the overall stress -strain curves under impact loading can be already obtained by SHPB measurement, a field measurement is still necessary because of heterogeneous fields within the foam specimen. High speed images together with the digital image correlation techniques is a complementary measurement to SHPB technique. They are not only used to evaluate the strain field of the foam specimen, but also to obtain the displacement history at the ends of pressure bars. By comparing this measurement with the ones from Hopkinson bars, it allows to correct the time synchronization error and to check the calibration of the strain/tension coefficients.

For the studied brittle polymeric foam, an overall rate sensitivity of $8 \%$ is found from quasistatic loading to impact loading with a speed of $20 \mathrm{~m} / \mathrm{s}$. Even though the equilibrium state (between forces of two bar/specimen) is reached rapidly in SHPB test, there is no homogeneous 
strain field. The DIC strain field measurement is the only mean to reveal this crushing mechanism like a honeycombs, i.e., the crush displacement (nominal strain) is mainly due to the enlargement of the very localized area/bands within the specimen and the uncollapsed part remains at a constant small strain.

\section{Acknowledgements}

The authors acknowledge the support of China Scholarship Council (CSC) as well as NSFC funding no11228206, and appreciate the kindness of EADS in supplying the specimens. 
[1] L. J. Gibson, M. F. Ashby, Cellular Solids: Structure and Properties, 2nd Edition, Cambridge University Press, Cambridge, 1997.

[2] N. Mills, C. Fitzgerald, A. Gilchrist, R. Verdejo, Polymer foams for personal protection: Cushions, shoes and helmets, Composites Science and Technology 63 (16) (2003) 23892400. doi:DOI10.1016/S0266-3538(03)00272-0

[3] H. Zhu, J. Knott, N. Mills, Analysis of the elastic properties of open-cell foams with tetrakaidecahedral cells, Journal of the Mechanics and Physics of Solids 45 (3) (1997) 319-\&.

[4] H. Zhu, N. Mills, J. Knott, Analysis of the high strain compression of open-cell foams, Journal of the Mechanics and Physics of Solids 45 (11-12) (1997) 1875-1904.

[5] V. Deshpande, N. Fleck, Multi-axial yield behaviour of polymer foams, Acta Materialia 49 (10) (2001) 1859-1866.

[6] L. Gong, S. Kyriakides, W. Jang, Compressive response of open-cell foams. part i: Morphology and elastic properties, International Journal of Solids and Structures 42 (5-6) (2005) 1355-1379. doi:DOI10.1016/j.ijsolstr.2004.07.023.

[7] L. Gong, S. Kyriakides, Compressive response of open cell foams - part ii: Initiation and evolution of crushing, International Journal of Solids and Structures 42 (5-6) (2005) 13811399. doi:DOI10.1016/j.ijsolstr.2004.07.024.

[8] R. Lacey, Response of several materials at intermediate strain rates, Paper From High Speed Testing 5 (1965) 99-109.

[9] R. Traeger, Physical properties of rigid polyurethane foams, Journal of Cellular Plastics 3 (9) (1967) 405-418.

[10] H. Schreyer, Q. Zuo, A. Maji, Anisotropic plasticity model for foams and honeycombs, Journal of engineering mechanics 120 (9) (1994) 1913-1930.

[11] O. Faruque, N. Liu, C. C. Chou, Strain rate dependent foam: Constitutive modeling and applications, SAE transactions 106 (5) (1997) 904-912.

[12] J. Zhang, N. Kikuchi, V. Li, A. Yee, G. Nusholtz, Constitutive modeling of polymeric foam material subjected to dynamic crash loading, International Journal of Impact Engineering 21 (5) (1998) 369-386. 
[13] J. D. Rehkopf, G. M. McNeice, G. W. Brodland, Fluid and matrix components of polyurethane foam behavior under cyclic compression, Journal of engineering materials and technology 118 (1) (1996) 58-62.

[14] D. A. Wagner, Y. Gur, S. M. Ward, M. A. Samus, Modeling foam damping materials in automotive structures, Journal of engineering materials and technology 119 (3) (1997) 279283.

[15] F. S. Chang, Y. Song, D. Lu, C. DeSilva, Unified constitutive equations of foam materials, Journal of engineering materials and technology 120 (3).

[16] J. Rinde, K. Hoge, Time and temperature dependence of the mechanical properties of polystyrene bead foam, Journal of Applied Polymer Science 15 (6) (1971) 1377-1395.

[17] J. Rinde, K. Hoge, Dynamic shear modulus of polystyrene bead foams, Journal of Applied Polymer Science 16 (6) (1972) 1409-1415.

[18] B. Song, W. Chen, D. J. Frew, Dynamic compressive response and failure behavior of an epoxy syntactic foam, Journal of composite materials 38 (11) (2004) 915-936.

[19] H. Zhao, G. Gary, J. Klepaczko, On the use of a viscoelastic split hopkinson pressure bar, International Journal of Impact Engineering 19 (4) (1997) 319-330.

[20] H. Zhao, G. Gary, Behaviour characterisation of polymeric foams over a large range of strain rates, International journal of vehicle design 30 (1) (2002) 135-145.

[21] H. Zhao, Testing of polymeric foams at high and medium strain rates, Polymer Testing 16 (5) (1997) 507-516.

[22] H. Zhao, I. Elnasri, S. Abdennadher, An experimental study on the behaviour under impact loading of metallic cellular materials, International Journal of Mechanical Sciences 47 (4-5) (2005) $757-774$.

[23] Y. Wang, G. Gioia, A. Cuitino, The deformation habits of compressed open-cell solid foams, Journal of Engineering Materials and Technology-Transactions of the Asme 122 (4) (2000) $376-378$.

[24] M. A. Sutton, J.-J. Orteu, H. Schreier, Image correlation for shape, motion and deformation measurements: basic concepts, theory and applications, Springer Publishing Company, Incorporated, 2009. 
[25] M. Grédiac, F. Hild, Mesures de champs et identification en mécanique des solides, Lavoisier, 2011.

[26] D. Zhang, X. Zhang, G. Cheng, Compression strain measurement by digital speckle correlation, Experimental Mechanics 39 (1) (1999) 62-65.

[27] Y. Wang, A. Cuitino, Full-field measurements of heterogeneous deformation patterns on polymeric foams using digital image correlation, International Journal of Solids and Structures 39 (13-14) (2002) 3777-3796.

[28] S. R. Heinz, J. S. Wiggins, Uniaxial compression analysis of glassy polymer networks using digital image correlation, Polymer Testing 29 (8) (2010) 925-932.

[29] A. Gilchrist, N. Mills, Impact deformation of rigid polymeric foams: experiments and fea modelling, International journal of impact engineering 25 (8) (2001) 767-786.

[30] P. Viot, F. Beani, J. Lataillade, Polymeric foam behavior under dynamic compressive loading, Journal of Materials Science 40 (22) (2005) 5829-5837. doi:DOI10.1007/s10853-005-4998-5

[31] S. Nemat-Nasser, W. Kang, J. McGee, W.-G. Guo, J. Isaacs, Experimental investigation of energy-absorption characteristics of components of sandwich structures, International journal of impact engineering 34 (6) (2007) 1119-1146.

[32] R. Othman, S. Aloui, A. Poitou, Identification of non-homogeneous stress fields in dynamic experiments with a non-parametric method, Polymer Testing 29 (5) (2010) 616-623. doi:DOI10.1016/j.polymertesting.2010.03.013.

[33] J. Liu, F. Lu, D. Fang, H. Zhao, Impact testing of cellular materials with field measurement-a review, International Journal of Protective Structures 2 (4) (2011) 401-416.

[34] H. Zhao, G. Gary, A three dimensional analytical solution of the longitudinal wave propagation in an infinite linear viscoelastic cylindrical bar. application to experimental techniques, Journal of the Mechanics and Physics of Solids 43 (8) (1995) 1335 - 1348.

[35] H. Zhao, G. Gary, On the use of shpb techniques to determine the dynamic behavior of materials in the range of small strains, International Journal of Solids and Structures 33 (23) (1996) $3363-3375$. 
[36] G. Besnard, F. Hild, S. Roux, "finite-element" displacement fields analysis from digital images: Application to portevin-le chatelier bands, Experimental Mechanics 46 (6) (2006) 789-803.

[37] F. Hild, S. Roux, Digital image correlation: from displacement measurement to identification of elastic properties-a review, Strain 42 (2) (2006) 69-80. 